\title{
Estratégias de relativização em Apurinã (Aruák) 1
}

\author{
Relativization strategies in Apurinã (Arawakan)
}

Resumo: O sistema de marcação e as várias funções envolvidas em orações relativas em Apurinã (Aruák) são descritos. Esse sistema envolve um complexo grupo morfêmico que, ao mesmo tempo em que pode ser segmentado em quatro morfemas distintos, funciona como uma construção morfológica marcando orações relativas na língua. Esse complexo morfêmico quadripartido envolve funções tipicamente associadas a orações relativas, tais como relação gramatical (sujeito e objeto), e outras menos típicas ou incomuns a funções gramaticalmente codificadas em relativizadores, como voz, polaridade, número e gênero. Uma breve relação é feita entre esse sistema de relativização em Apurinã e a tipologia sobre o fenômeno das orações relativas.

Palavras-chave: Orações relativas. Tipologia. Apurinã. Aruák.

Abstract: A system of relative clauses is described for the Apurinã language (Arawakan). The system consists of a complex morphemic cluster that, although it can be segmented into four distinct morphemes, acts as a morphological construction marking relative clauses in this language. This complex four-part construction encodes functions typically associated with relative clauses in other languages such as grammatical relations (subject and object), and functions atypical for the grammatical encoding of relative clauses such as voice, polarity, number and gender. A brief attempt to situate the system in terms of the typology of relative clause is also presented.

Key words: Relative clauses. Typology. Apurinã. Arawakan.

I Mahidol University. Professor visitante. Universidade Federal do Pará. Professor adjunto. Belém, Pará, Brasil (sfacundes@gmail.com).

1 Este artigo foi concluído quando o autor estava licenciado da Universidade Federal do Pará, atuando como professor visitante na Mahidol University.

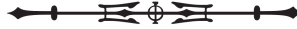




\section{INFORMAÇÕES PRELIMINARES}

Apurinã (sãkiretxi 'língua') é uma língua do ramo Purus da família Aruák, (segundo a classificação baseada em retenções lexicais compartilhadas proposta por Payne (1993); consultar também Aikhenvald (1999), Facundes (2002, no prelo), e Facundes e Brandão (no prelo)); é falada em mais de 20 comunidades espalhadas principalmente ao longo de afluentes do rio Purus, no sudeste do estado do Amazonas. Suas comunidades mais ao sul encontram-se nos Km 124 e 45 da BR-317, que liga Rio Branco a Boca do Acre; a comunidade mais ao norte encontra-se na foz do rio Jatuarana, próximo ao município de Manacapuru, no estado do Amazonas. Boa parte de sua população vive na periferia das cidades de Pauini, Lábrea e Tapauá, além de outras pequenas cidades ribeirinhas da Bacia do Purus. Há falantes de Apurinã vivendo também em aldeias de grupos de línguas tupí em Rondônia, assim como junto aos jamamadi e paumari no Purus. Os dados nos quais se basearam os resultados da pesquisa aqui apresentada têm sido coletados e analisados pelo próprio autor desde 1990, quando este realizou a sua primeira visita às comunidades apurinã. Uma apresentação preliminar da gramática dessa língua, assim como informações socioculturais sobre as comunidades que a falam, podem ser encontradas em Facundes (2000b).

Apesar de suas características polissintéticas, o que faz com que muitas das funções geralmente associadas a processos sintáticos sejam expressas morfologicamente, Apurinã apresenta várias operações que entram em ação na combinação entre orações simples para produzir frases complexas ou combinar orações independentes. Relativização é uma dessas operações e será o foco deste trabalho. A Figura 1 contextualiza de forma representativa orações relativas como um dos processos envolvidos na combinação de orações na língua.

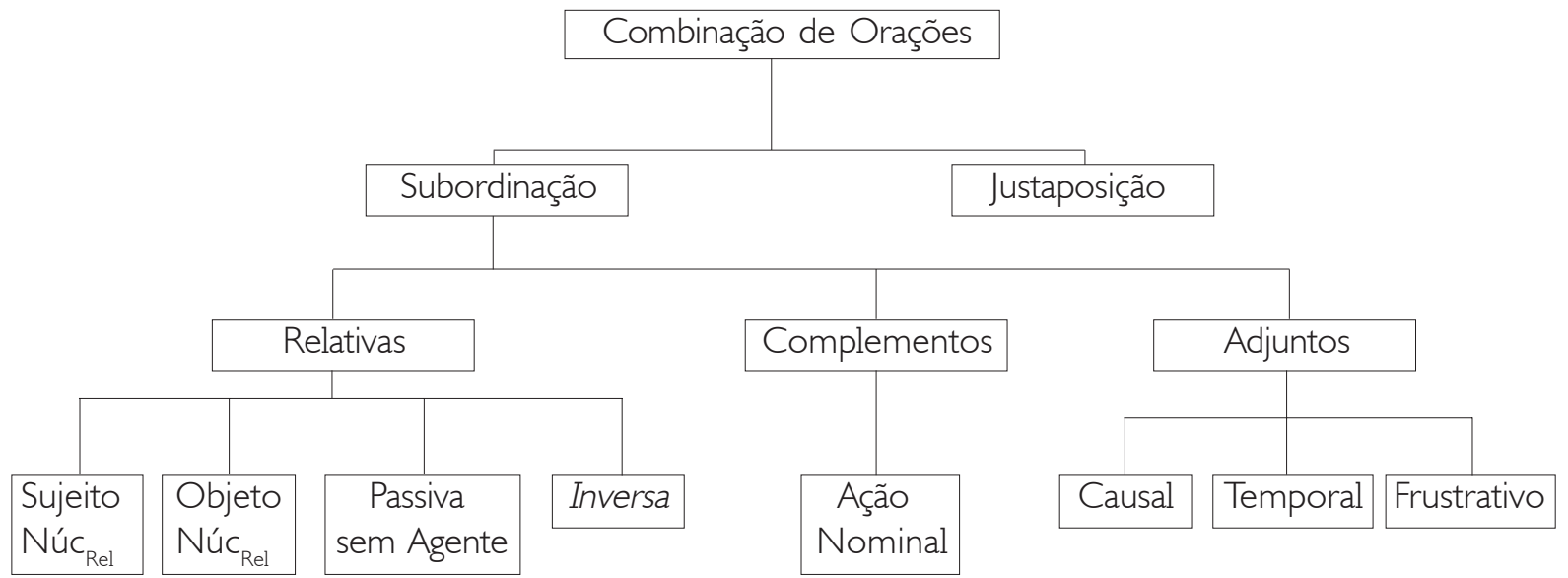

Figura 1. Processos envolvidos na combinação de orações (adaptado de Facundes, 2000b)

A primeira menção sobre as marcas relativizadoras em Apurinã foi feita por Pickering (1977); contudo, sem uma descrição sobre as diversas propriedades do sistema.

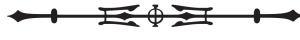




\section{DEFINIÇÃO DE ORAÇÕES RELATIVAS E DOS PARÂMETROS USADOS NO ESTABELECIMENTO DE SUA TIPOLOGIA²}

A descrição do fenômeno segue aqui a tradição funcional-tipológica já muito bem estabelecida na literatura lingüística sobre a descrição gramatical e sobre o estabelecimento dos tipos gramaticais encontrados nas línguas do mundo, como pode ser confirmado em Greenberg (1966), Comrie (1981; 1989), Givón (1989; 1990), Dryer (1986), entre outros. Essa abordagem funcional-tipológica permite revelar os fatos cruciais da gramática da língua que não podem ser ignorados por nenhum modelo teórico da gramática e que, portanto, podem ainda, mais adiante, receber um tratamento teórico (formal ou não) mais específico. Fazendo uso dessa abordagem funcional-tipológica, algumas noções serão empregadas como procedimentos heurísticos que têm se mostrado bastante úteis como parte do processo de descoberta. Este é o caso quando, ao se fazer referência às relações relevantes entre o predicado e os seus argumentos, utilizam-se as notações A, S, P, T e R, com as seguintes significações: S corresponde ao único argumento de um verbo intransitivo; A corresponde ao argumento que mais se aproxima de agente; $\mathrm{P}$ (utilizado ao invés de $O$ para não ser confundido com a relação gramatical objeto) corresponde ao argumento que mais se correlaciona com o paciente; $T$ corresponde ao argumento de um verbo ditransitivo que se desloca no espaço físico ou metafórico; e R corresponde ao argumento de um verbo ditransitivo que funciona como recebedor ou beneficiário da ação expressa pelo predicado. Assim, pode-se ilustrar tais notações em português conforme exposto no exemplo (1):

$$
\text { S } \vee
$$

a. $\quad$ Ele anda.

$$
\text { A P V }
$$

b. Ele a comprou.

$$
\text { A } T \vee \quad R
$$

c. Ele a deu para mim.

Essas notações permitem uma visualização clara do mapeamento entre certos sintagmas nominais e os macro-papéis sintático-semânticos mais prototípicos, ou seja, aqueles que podem ser abstraídos ao comparar os fatores transitividade e papel semântico prototípico, antes que se possa observar o seu comportamento morfossintático e determinar o seu status como relação gramatical (sujeito, objeto etc.) e o padrão em torno do qual tais relações gramaticais organizam-se, ou seja, com alinhamentos nominal-acusativo, ergativoabsolutivo etc. No Português Brasileiro (no registro culto), por exemplo, as formas pronominais não possessivas permitem o agrupamento de A com S (ambos usam os pronomes "eu, tu, ele...") e de T com P (ambos usam "me, te, o/a..."), ficando R sozinho em outro grupo (pois faz uso de "mim, ti, ele..." quando usado com preposições), produzindo evidência em favor das noções sintáticas sujeito, objeto direto e objeto indireto nessa língua.

Em Apurinã, as estratégias de relativização envolvem complexos recursos morfossintáticos, dos quais aquele presente na oração relativa $\left(O r_{\text {Rel }}\right)$ em (2) é uma ilustração típica: nessa oração o núcleo relativo (Núc $\left.c_{\text {Rel }}\right)$ é

\footnotetext{
2 Por restrições de espaço, este trabalho discute apenas orações relativas com predicatos verbais. Há diferenças significativas entre predicados verbais e não-verbais em frases complexas na língua.
}

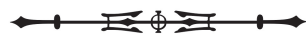


sytu 'mulher', que é modificado pela Or ${ }_{\text {Rel }}$ kyky karuta-karu 'que feriu o homem’3 (elementos coreferenciais são sublinhados). $\mathrm{Na} \mathrm{Or}_{\mathrm{Rel}}$ em (2) há a forma presa -karu, a primeira de uma série de formas

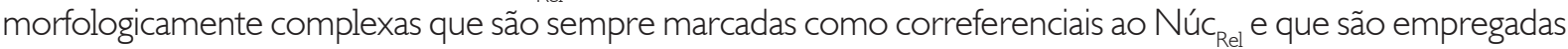
no verbo. A frase complexa em (3) é análoga, sendo que o verbo da oração matriz é transitivo.

S/A $\quad\left[\begin{array}{llll}P & \text { V-REL.A }\end{array}\right] \mathrm{Or}_{\mathrm{Rel}} \quad \mathrm{V}$ sytu kyky karuta-karu apu-pe mulher homem ferir-REL.F.A chegar-PFTV

'A mulher que feriu o homem chegou.'

\begin{tabular}{|c|c|c|c|}
\hline $\mathrm{A} / \mathrm{A}$ & {$[P$} & V-REL.A JOr $r_{\text {Rel }}$ & \\
\hline $\begin{array}{l}\text { sytu } \\
\text { mulher }\end{array}$ & $\begin{array}{l}\text { kyky } \\
\text { homem }\end{array}$ & $\begin{array}{l}\text { karuta-karu } \\
\text { ferir-REL.F.A }\end{array}$ & $\begin{array}{l}\text { ytyka-nu } \\
\text { ver-VBLZ-3SG.O }\end{array}$ \\
\hline
\end{tabular}

A frase complexa em (2) pode ser interpretada como a equivalente proposicional de duas orações simples como aquelas em (4a) e (4b), enquanto que a frase complexa do exemplo (3) pode ser interpretada como a equivalente proposicional de duas orações simples como as apresentadas em (4a) e (4c).

$\begin{array}{llll}\text { (4) a. } & \begin{array}{l}\text { kyky } \\ \text { homem }\end{array} & \text { sytu } & \text { karuta } \\ \text { 'A mulher feriu } & \text { ferir }\end{array}$
$\mathrm{S} \quad \mathrm{V}$
b. $\quad$ sytu apu-pe
mulher chegar-PFTV
'A mulher já chegou.'
A $\quad$ - $-\mathrm{P}$
c. sytu ytyka-nu
mulher ver-VBLZ-1SG.P
'A mulher me viu.'

Ou seja, ao receber a forma -karu as orações em (4b) e (4c) são 'nominalizadas' (ou seja, adquirem o status de um SN), tornando-se, cada uma delas, uma oração relativa encaixada como argumento $S$ ou A na oração matriz. Estas características também se aplicam às demais formas relativizadoras descritas neste trabalho.

Em contraste aos exemplos (2) e (3), em (5) há uma outra frase complexa em que sytu novamente é o Núc $\mathrm{Rel}_{\text {el }}$ e kyky karuta-kytu 'ferida pelo homem' é a Or ${ }_{\text {Rel' }}$

3 Na notação S/A, S corresponde ao status do argumento na oração matriz e A corresponde ao status do argumento na oração relativa. Portanto, sytu é S na oração matriz, mas A na oração relativa. Em S/O, S corresponde ao status do argumento na matriz e $\mathrm{O}$ ao status do argumento na oração relativa. As demais combinações são análogas.

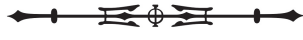




\section{(5) sytu kyky karuta- $\underline{\text { kytu }}$ apu-pe \\ mulher homem ferir-REL.F.P chegar-PFTV \\ 'A mulher ferida pelo homem já chegou.'}

Enquanto que em (2) e (3) o marcador pronominal relativo é -kary, na frase (5) a forma pronominal empregada no verbo é -kytu. O marcador -karu é usado quando o Núc $c_{\text {Rel }}$ é um argumento A ou S na $\mathrm{Or}_{\mathrm{Rel}}$; já -kytu é usado quando o Núc $\mathrm{Rel}_{\text {é }}$ um argumento $\mathrm{P}$ da $\mathrm{Or}_{\text {Rel }}$ Portanto, os marcadores pronominais relativos codificam diretamente a distinção gramatical entre sujeito (A e S) e objeto (P), cuja oposição sintática é independentemente motivada na língua (FACUNDES, 2000b).

A próxima frase (6a) ilustra casos em que o Núc $c_{\text {Rel' }}$ sytu, funciona como o argumento beneficiário (R) do verbo da $\mathrm{Or}_{\mathrm{Rel}}$ nhipuku-ry ny-syka-kytu 'para quem dei a comida'. Na frase (6b) é nhipuku-ry 'comidaDESPOS' que é o Núc Rel' portanto, demonstrando que as expressões do argumento tema (T, neste caso, 'aquilo que é dado') e R seguem a mesma estratégia de relativização daquela do argumento P. $\bigcirc$ alinhamento dos argumentos verbais nas orações relativas é representado na Figura 2.

$$
\mathrm{S} / \mathrm{R} \quad[\mathrm{T} \quad \mathrm{A}-\mathrm{V}-\mathrm{REL} . \mathrm{R}] \mathrm{Or}_{\mathrm{Rel}} \mathrm{V}
$$
a. $\underline{\text { sytu }}$
nhipuku-ry
ny-syka-kytu sy-pe
mulher comida-DESPOS 1SG-dar-REL.F.R ir-PFTV

'A mulher para quem dei a comida já foi.'
$\mathrm{S} / \mathrm{T}$
$[R$
b. nhipuku-ry
sytu-muni
A-V-REL.T ]Or ${ }_{\text {Rel }} \quad \mathrm{V}$
comida-DESPOS mulher-META 1SG-dar-REL.M.T ser.bom-PRED
'A comida que dei para a mulher estava gostosa.'

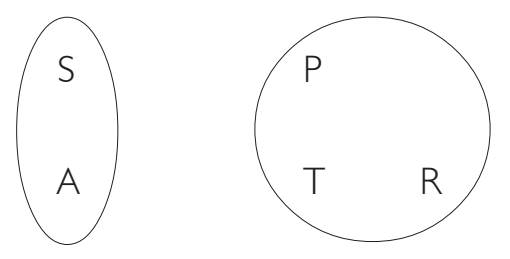

Figura 2. Alinhamento dos argumentos verbais nas orações relativas.

Portanto, doravante pode-se descrever as demais propriedades gramaticais das $\mathrm{Or}_{\text {Rel }}$ na língua, tratando-se A e $\mathrm{S}$ como sujeito e P, T e R como objeto - ao menos no que diz respeito ao alinhamento dos macro-papéis sintático-semânticos nas $\mathrm{Or}_{\mathrm{Rel}}$ S Sujeito será representado no resto deste trabalho por $\mathrm{S}$ e objeto será representado por $\mathrm{O}$. Contudo, na descrição de alguns dados, será importante identificar a associação entre o papel semântico de um argumento e o status sintático de sua expressão formal; nesses casos, as distinções entre os argumentos $\mathrm{A}, \mathrm{S}$ e $\mathrm{P}$ serão mantidas.

Em frases complexas contendo uma $\mathrm{Or}_{\text {Rel' }}$ somente o papel gramatical do $N$ úc $_{\text {Rel }}$ na $\mathrm{Or}_{\text {Rel }}$ é relevante para determinar o tipo de marcador relativo. O papel gramatical do Núc $c_{\text {Rel }}$ na oração matriz não interfere na

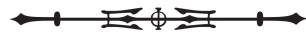


marcação formal de relativização em Apurinã. Isso pode ser observado nos exemplos (2), (5) e (6a), comparados às frases em (7). Nas frases em (2), (5) e (6a), o Núc Rel é sujeito na matriz e ambos os marcadores relativos -karu e -kytu são empregados. Em (7), a presença do marcador pronominal de objeto, -ru, em $n$-apuku-ru, indica que o Núc $c_{\text {Rel }}$ sytu é objeto na matriz e, novamente, ambos, -karu e -kytu, são empregados:

$\begin{array}{lllll}\text { (7) } \quad \begin{array}{ll}\text { S-V-O } \\ \text { n-apuka- } \underline{\text { ru }}\end{array} & \mathrm{O} / \mathrm{S} & {[\mathrm{O}} & \text { V-REL.S }] \\ & \text { 1SG-achar-3F.O } & \text { kyky } & \text { mulher } \\ & \text { homem } & \text { mulher-REL.F.S }\end{array}$

'Eu achei a mulher que matou o homem.'
$\mathrm{s}-\mathrm{V}-\mathrm{O}$
$\mathrm{O} / \mathrm{O} \quad[\mathrm{S}$
V-REL.O ]
b. n-apuka- $\underline{\mathrm{ru}}$
sytu kyky
uka- $\underline{k y t u}$
1SG-achar-3F.O mulher homem matar-REL.F.O
'Eu achei a mulher morta pelo homem.'

As orações relativas não restritivas seguem o mesmo padrão formal das restritivas. Na frase (8), kayãparu 'Kayãparu' é o Núc $c_{\text {Rel }}$ que é modificado pela $O r_{\text {Rel }}$ (nu-sytu-re inha-karu 'que é minha mulher'). O Núc $c_{\text {Rel }}$ kayãparu, como um nome próprio, é um SN que é totalmente especificado e identificado e a informação expressa pela $\mathrm{Or}_{\text {Rel }}$ não é pressuposta. Portanto, $\mathrm{Or}_{\text {Rel }}$ não restritivas fazem uso dos mesmos relativizadores que as $\mathrm{Or}_{\text {Rel }}$ restritivas.

$\begin{array}{llll}\text { S/S } & {[\text { PN }} & \text { V-REL.S }] & \text { V } \\ \text { kayãparu } & \text { ny-sytu-re } & \text { inha-karu } & \text { apu-pe } \\ \text { Kayãparu } & \text { 1SG-mulher-POSSDO } & \text { ser-REL.F.S } & \text { chegar-PFTV } \\ \text { 'Kayãparu, minha esposa, já chegou.' } & & \\ \text { (lit.: 'Kayãparu, que é minha esposa, já chegou.') } & \end{array}$

Em um quadro geral, representado preliminarmente na Tabela 1 (e revisado mais adiante), é possível afirmar que as estratégias de relativização em Apurinã organizam-se em torno de um sistema gramatical regido por quatro tipos de informações codificadas no relativizador pronominal: sujeito, objeto, ausência de agente ou presença de terceira pessoa pronominal atuando em um participante do discurso. Ainda que o sistema admita alguns casos de sincretismos, tais marcadores variam ainda segundo a pessoa gramatical, o gênero, o número e a polaridade verbal. $\bigcirc$ resto deste trabalho descreverá sucintamente cada um dos quatro tipos gerais e seus subtipos.

Tabela 1. Sistema de marcadores pronominais relativos

\begin{tabular}{|c|c|c|c|c|c|c|c|}
\hline & & \multicolumn{2}{|c|}{ Sujeito } & \multirow[t]{2}{*}{ Objeto } & \multicolumn{2}{|c|}{ Relativa sem Agente } & \multirow{2}{*}{$\begin{array}{l}3>1,2 \mathrm{PRO} \\
3>1,2 \mathrm{PROCL}\end{array}$} \\
\hline & & Positiva & Negativa & & Positiva & Negativa & \\
\hline \multirow[t]{2}{*}{ SG. } & $M$ & -kary & -katy & -kyty & -kury & -kuty & -keri \\
\hline & $\mathrm{F}$ & -karu & -katu & -kytu & -kuru & -kutu & -keru \\
\hline PL. & $M / F$ & -kany & -kyny & -kuny & -kenu & & \\
\hline
\end{tabular}

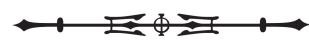




\section{MARCAÇÃO DE GÊNERO E NÚMERO NA OR}

Comparando o $\mathrm{Núc}_{\mathrm{Rel}}$ na $\mathrm{Or}_{\mathrm{Rel}}$ do exemplo (2) ao Núc $\mathrm{Rel}_{\text {Re }}$ na $\mathrm{r}_{\text {Rel }}$ do exemplo (9), observa-se que a única diferença entre os dois é o gênero. Em (2), o Núc $c_{\text {Rel }}$ é sytu 'mulher', que é modificado pela $O r_{\text {Rel }} k y k y$ karuta-karu 'que feriu o homem'; já em (9), o Núc $c_{\text {Rel }}$ é kyky 'homem', que é modificado pela Or $r_{\text {Rel }}$ sytu karutakary 'que feriu a mulher'. Ou seja, o marcador nominal relativo concorda em gênero com o Núc $c_{\text {Rel }}$ da frase e essa marcação é feita através da alternância entre as vogais finais /u/ e /y/. Em (10) -kyty é o marcador pronominal relativo de objeto que concorda com o Núc $c_{\text {Rel }}$ masculino kyky. Essa frase pode ser comparada ao exemplo (5) na qual a contraparte feminina -kytu é empregada, concordando com o Núc $c_{\text {Rel }} s y t u$.

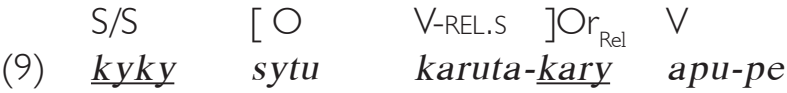
homem mulher ferir-REL.M.S chegar-PFTV

'O homem que feriu a mulher já chegou.'

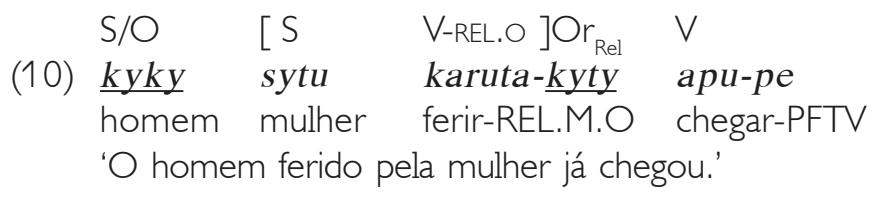

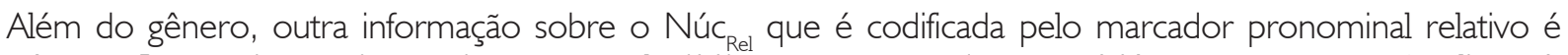
número. Isso pode ser observado no exemplo (11): -kany concorda com o Núc Rel $_{\text {s }}$ sytu-waku-ru 'mulheres', sujeito em (11a); em constraste, -kyny concorda com o Núc $c_{\text {Rel }} s y t u$-waku-ru, objeto em (11b):

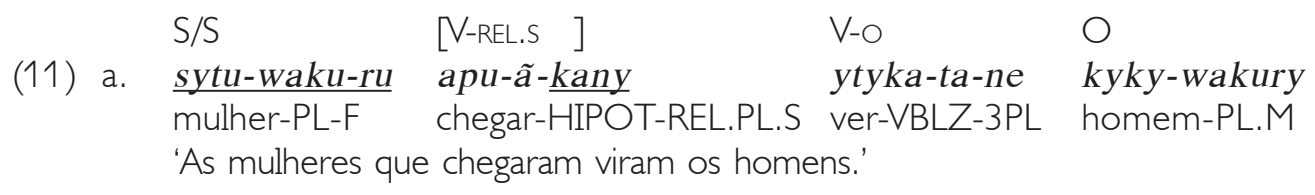
$\mathrm{O} / \mathrm{O}$
$[\mathrm{s}-\mathrm{V}-\mathrm{REL} . \mathrm{P}]$
S V
b. sytu-waku-ru n-ytyka-ta-kyny kuna pitha ytyka-ta mulher-PL-F 1SG-ver-VBLZ-REL.PL.S não 2SG ver-VBLZ
'As mulheres que eu vi, tu não viste.'

As ocorrências do exemplo (12) demonstram que os marcadores relativos do plural não codificam a distinção de gênero (tanto na função de sujeito como de objeto); isto é, as formas relativizadoras -kany e -kyny não distinguem gênero.

\begin{tabular}{|c|c|c|c|c|}
\hline (12) a. & $\begin{array}{l}\text { S/S } \\
\frac{\text { kyky-wakury }}{\text { homem-PL.F }}\end{array}$ & $\begin{array}{l}\text { [V-REL.S ] } \\
\text { apu-ã-kany } \\
\text { chegar-HYPOTH-REL.PL.S }\end{array}$ & $\begin{array}{l}\text { V-O } \\
\text { ytyka-ta-ne } \\
\text { ver-VBLZ-3M.O }\end{array}$ & $\begin{array}{l}\text { O } \\
\text { sytu-waku-ru } \\
\text { mulher-PL-F }\end{array}$ \\
\hline
\end{tabular}
$\mathrm{O} / \mathrm{O}$
$[$ S-V-REL.P ]
b. kyky-waku-ry n-ytyka-ta-kyny
homem-PL-F ver-VBLZ-REL.PL.S
'Os homens que eu vi, tu não viste.'
kuna pitha ytyka-ta
não 2SG ver-VBLZ

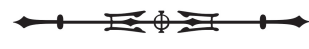




\section{MARCAÇÃO DA POLARIDADE VERBAL NA OR REL}

Além de relação gramatical, gênero e número, os marcadores pronominais relativos também codificam a distinção entre as polaridades positiva e negativa. Tal distinção pode ser observada ao se comparar as frases (2) e (9) àquelas dadas em (13). Na presença do marcador negativo ma-, formador de formas verbais descritivas (verbos intransitivos que admitem marcadores pronominais de objeto e não de marcadores pronominais de sujeito), o marcador pronominal relativo adicionado ao verbo, exemplo (13a), assume uma forma distinta daquelas vistas até aqui. A frase (13b) é análoga. Nos dois casos a variação na forma que corresponde à marcação da oposição entre polaridades é a alternância entre $-r$ e $-t$, sendo que a primeira das duas consoantes marca a polaridade positiva e a segunda marca a polaridade negativa:
$\mathrm{S} / \mathrm{S}$
a. $\quad \underline{s y t u}$
[NEG-V-REL.S
mulher
ma-kirãta-rewa-ta-katu
$\mathrm{V}$
mireka
acordar
'A mulher que não ronca está acordada.'
$\begin{array}{llll} & \mathrm{S} / \mathrm{S} & {[\mathrm{NEG}-V-\mathrm{REL} . \mathrm{S} \quad]} & \mathrm{V} \\ \text { b. } & \text { kyky } & \text { ma-kirãta-rewa-ta-katy } & \text { mireka } \\ & \text { homem } & \text { PRIV-roncar-INTR-VBLZ-REL.NEG.F } & \text { acordar }\end{array}$
'O homem que não ronca está acordado.'

\section{MARCAÇÃO DE PASSNA SEM AGENTE NA OR}

Algumas $\mathrm{Or}_{\text {Rel }}$ com verbos transitivos ocorrem obrigatoriamente sem o argumento A e o argumento P é expresso como S. No sentido em que o sujeito dessas $O r_{\text {Rel }}$ codifica sistematicamente o paciente, essa Or $r_{\text {Rel }}$ assemelha-se a uma oração passiva, exceto que o agente é obrigatoriamente omitido,daí associá-la ao tipo de passiva comumente denominada passiva sem agente.

Tomando como ponto de partida o significado proposicional das frases simples em (14a-b), a frase complexa (15) pode ser construída. Em (14a) o morfema - $\tilde{V}$ ka funciona, em alguns dos seus usos, como um marcador de passiva (FACUNDES, 2000b) e, em (14b), há uma frase simples transitiva ${ }^{4}$. Nos contextos relevantes, os referentes dos sujeitos dessas duas frases são co-referenciais e o sentido proposicional total pode ser expresso através da frase complexa (15a). Essa frase é analisável como consistindo da oração matriz n-atama-ta-ry 'Eu o vi', precedendo o Núc $c_{\text {Rel }}$ manhiti 'veado' mais a Or $r_{\text {Rel }}$ nhika-kury 'que foi comido'. Como visto em (15a), construções relativas passivas sem agente requerem o uso do marcador pronominal relativo -kury. Já a frase complexa em (15b) não é gramatical porque o agente na $O r_{\text {Rel }}$ é explicitamente mencionado:

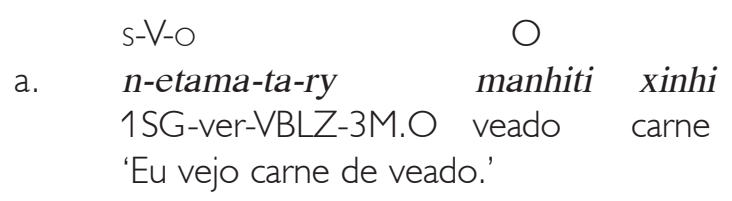

\footnotetext{
4 Em algumas variedades da língua Apurinã esse marcador de passiva assume a forma - $k a$, ou seja, a vogal imediatamente precedente é nasalizada, mas não prolongada.
}

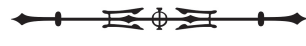




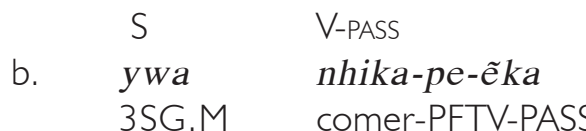

'Ele foi comido.'
$\mathrm{s}-\mathrm{V}-\mathrm{O}$
a. n-etama-ta-ry
$\mathrm{O} / \mathrm{O}$
[V-REL.PASS $] \mathrm{Or}_{\mathrm{Re}}$
1SG-ver-VBLZ-3M.O veado carne comer-REL.M.PASS
'Eu vejo a carne do veado que estão comendo.'
$\mathrm{s}-\mathrm{V}-\mathrm{O}$
b. * * n-etama-ta-ry
$\mathrm{O} / \mathrm{O} \quad \mathrm{S}$
1SG-ver-VBLZ-3MO veado carne $3 \mathrm{PL}$
$\begin{array}{ll}\text { nynuwa } & \text { [V-REL.PASS }] \text { Or } \\ \text { nhika- } \text { Rury }\end{array}$
comer-REL.M.PASS

1SG-ver-VBLZ-3M.O veado carne 3PL

(Eu vejo a carne do veado comida pela onça.)

Os dados expostos no exemplo (16) são análogos e ilustram as contrapartes feminina positiva e negativa do marcador pronominal relativo, -kuru e -kutu, respectivamente:
a.

s-V-O
$n$-apuka-ru
1SG-ver-3F.O
$\mathrm{O} / \mathrm{S}$
$\underline{x i r i p i-t x i}$
[ V-REL.PASS $] \mathrm{Or}_{\text {Rel }}$
1SG-ver-3F.O flecha-DESPOS quebrar-REL.F.PASS
'Eu achei a flecha que foi quebrada.'
(lit.: 'Eu achei uma flecha que tinha sido quebrada.')
s-V-O
$\mathrm{O} / \mathrm{S}$
b. n-apuka-ru
$\underline{x i r i p i-t x i}$
[ NEG-V-REL.PASS $] \mathrm{Or}_{\text {Rel }}$
1SG-achar-3F.O flecha-DESPOSS PRIV-quebrar-REL.F.NEG.PASS
'Eu achei uma flecha que não foi quebrada.'

MARCAÇÃO DE 'INVERSÃO' 5 NA OR ${ }_{\text {REL }}$

Há ainda um último tipo de $\mathrm{Or}_{\text {Rel }}$ que recebe marcadores pronominais relativos diferentes daqueles vistos até aqui. Tais marcadores consistem das formas $-k^{2} r^{6}{ }^{6}$ e -keru, masculino e feminino, respectivamente, para os quais distinção de número não foi atestada. A descrição das propriedades (um tanto complexas) desses marcadores requer menção do seu comportamento distribucional e funcional. Em termos distribucionais, esses marcadores são empregados em verbos transitivos em $\mathrm{Or}_{\text {Rel }}$ que contêm um sujeito codificado (explicitamente) por uma forma pronominal independente referindo-se à primeira ou segunda pessoa do singular, ou como qualquer um dos marcadores pronominais (formas pronominais presas). Em

5 O termo 'inversão' e seus derivativos são usados neste trabalho para estabelecer a relação tipológica com o fenômeno de mesmo nome atestado em outras línguas, porém, sem a pretensão de implicar tratarem-se exatamente do mesmo fenômeno.

6 Na variedade da comunidade Vista Alegre, -kery assume a a forma -kiri. A vogal 'e' passa a [i], causando a assimilação (descontígua) completa da vogal final. Alguns dos dados apresentados nesta seção pertencem a essa comunidade.

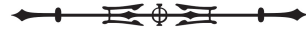


termos funcionais, -kery e -keru 'invertem' o papel semântico normalmente associado ao sujeito e objeto expressos através dessas formas pronominais (livres ou presas). Para ilustrar tais propriedades, tomemos como ponto de referência as frases simples do exemplo (17), onde a interpretação relevante é uma em que os sujeitos de ambas as frases são co-referenciais. Se essas duas frases forem combinadas em uma frase complexa para produzir o mesmo sentido proposicional, o resultado é que (17a) seria encaixada em (17b) como uma $\mathrm{Or}_{\text {Rel }}$ marcada com -kery, produzindo a frase (18):

$$
\text { P a-V }
$$

(17) a. kyky n-/p-/a-/h-etama-ta homem 1SG/3M/2SG/1 PL/2PL-ver-VBLZ

'Eu/Tu/Nós/Nocês vi/viste/vimos/viram o homem.'

S $\quad \mathrm{a}$

b. kyky apu-pe

homem chegar-PFTV

'O homem já chegou.'
S/A
$[\mathrm{p}-\mathrm{V}$-REL.INV
kyky
n-/py-/a-/h-auky-ta-kiri
]$_{\text {Orel }} \quad \mathrm{V}$
homem 1SG/2SG/1 PL/2PL-ver-REL.M.INV
apu-pe
chegar-PFTV

'O homem que me/te/nos/vos viu já chegou.'

Na frase (18), kyky é o Núc $c_{\text {Rel' }} n$-/py-/a-/h-atamatakiri 'que me/te/nos/vos viu' é a $\mathrm{Or}_{\mathrm{Rel}}$ ' e apu-pe 'já chegou' é o verbo da oração matriz. Observa-se que o sentido proposicional da frase complexa (18) é diferente do sentido das frases (17a) e (17b): o marcador pronominal de sujeito $n$-/py-/a-/h-, que era associado ao argumento semântico agentivo (típico de A) na oração simples em (17a), associa-se ao argumento paciente (típico de P) na $\mathrm{Or}_{\text {Rel }}$ em (18); além disso, a forma kyky 'homem' que se associara ao paciente em (17a), associa-se ao agente na $\mathrm{Or}_{\text {Rel }}$ em (18). Portanto, o uso das notações S/A (argumento $S$ na oração matriz/ argumento A na $\left.\mathrm{Or}_{\mathrm{Re}}\right)$ sobre kyky, e $p$ - sobre $n$-/py-/a-/h- no exemplo (18) reflete os macro-papéis sintáticosemânticos que estariam associados a essas formas não fosse a presença do marcador pronominal relativo de Or $r_{\text {Rel }}$ inversa, -kiri.

Os dados dos exemplos (19) e (20) são análogos e ilustram o mesmo processo de relativização realizado na presença de formas pronominais independentes funcionando como argumento da $\mathrm{Or}_{\text {Rel' }}$, neste caso com o Núc $c_{\text {Rel }}$ do gênero feminino:

P A $\quad \mathrm{V}$

a. sytu nuta/pita auky-ta

mulher 1SG/2SG ver-VBLZ

'Eu/Tu vi/viste a mulher.'

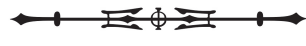




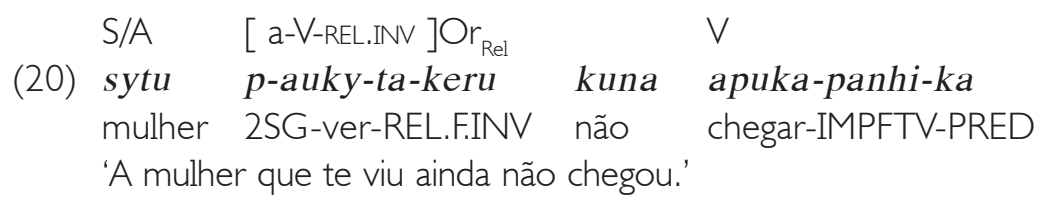

Finalmente, as ocorrências expostas no exemplo (21) demonstram o fato de o uso de O $r_{\text {Rel }}$ 'inversas' restringir-se às instâncias em que o sujeito da $O r_{\text {Rel }}$ é um pronome independente de primeira ou segunda pessoa ou um marcador pronominal. A frase (21a) não é gramatical porque o sujeito da $\mathrm{Or}_{\text {Rel }}$ é expresso na forma de um nome; já em (21b) o sujeito da $\mathrm{Or}_{\text {Rel }}$ está expresso na forma de um pronome independente na terceira pessoa do singular:

\begin{tabular}{|c|c|c|c|c|}
\hline 1) & $\begin{array}{l}\text { S/A } \\
* \text { kyky }\end{array}$ & & $\begin{array}{l}\text { V-REL.INV JOr }_{\text {Rel }} \\
\text { karuta-kiri }\end{array}$ & $\begin{array}{l}\vee \\
\text { apu-pe }\end{array}$ \\
\hline & homem & $\begin{array}{l}\text { mulher } \\
\text { que feriu }\end{array}$ & $\begin{array}{l}\text { ferir-REL.M.INV } \\
\text { mulher já chegou. }\end{array}$ & P I \\
\hline
\end{tabular}
S/A
b. $\quad *$ kyky
man

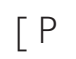
uwa/ywa/ata/hite/ynawa
1PL/3SG.F/3SG.M/1PL/2PL/3PL
V-rel.inv ]Or $r_{\text {Rel }} \vee$
karuta-kiri
apu-pe
( $O$ homem que a/o/nos/te feriu já chegou.)
ferir-REL.M.INV
chegar-PFTV

$\mathrm{O}$ uso das $\mathrm{Or}_{\text {Rel }}$ inversas é reminiscente de sistemas inversos de línguas algonquianas da América do Norte (FOLEY; VAN VALN, 1985) de duas maneiras: primeiramente, os morfemas presos normalmente associados ao argumento A passam a ser associados ao argumento P; em segundo lugar, uma das condições para o uso desse tipo de $\mathrm{Or}_{\text {Rel }}$ é que o argumento A ocorra na terceira pessoa e o argumento P ocorra na primeira ou segunda pessoa, portanto, seguindo parcialmente uma hierarquia de pessoa.

$\mathrm{OR}_{\text {REL }}$ SEM NÚC REL $_{\text {. }}$

Em todos os tipos de $\mathrm{Or}_{\text {Rel }}$ descritos até aqui, o Núc $\mathrm{Rel}_{\text {Rel }}$ modificado pela $\mathrm{Or}_{\text {Rel }}$ está presente. Em um sistema de relativização com tantas especializações distintas, não seria surpresa encontrar $\mathrm{Or}_{\text {Rel }}$ sem Núc $\mathrm{Rel}_{\text {el }}$, de fato, elas existem, conforme demonstram as ocorrências do exemplo (22). Em (22a), a Or $\mathrm{r}_{\text {Rel }}$ é sytu uka-kary 'quem/o que matou a mulher', e o sujeito é codificado apenas pelo marcador pronominal relativo de sujeito, -kary. Em (22b), a Or $r_{\text {Rel }}$ é a-nhika-kyty 'o que nós comemos', e o objeto é codificado pelo marcador pronominal relativo de objeto -kyty. As frases (23) a (25) ilustram os demais tipos de $\mathrm{Or}_{\text {Rel }}$ que também podem prescindir do $\mathrm{Nú}_{\mathrm{Rel}}$.

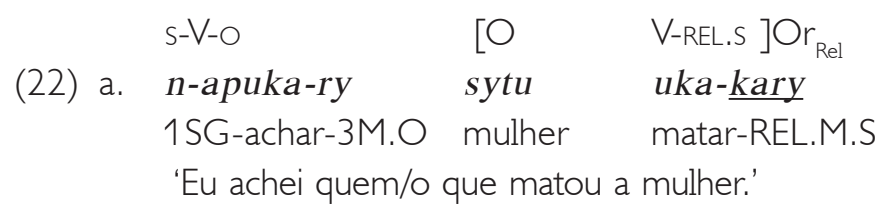

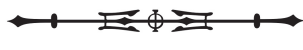


[S-V-REL.P $\quad \mathrm{Or}_{\text {Rel }} \quad \mathrm{V}-\mathrm{O}$

b. a-nhika-kyty

hareka-pyty-ry

1 PL-comer-REL.M.O ser.bom-ÊNF-3M.O

'O que nós comemos é bom demais.'

(23) ma-miteka-kati-nhi upỹpe

PRIV-correr-REL.M.S-AFET morrer

'O que/Quem não correu morreu.'

$\mathrm{s}-\mathrm{V}-\mathrm{O}$

p-apuka-ry

$[\mathrm{s}-\mathrm{V}$-REL.INV $] \mathrm{Or} \mathrm{r}_{\text {Rel }}$

2SG-achar-3M.O 1SG-ver-VBLZ-REL.M.S

'Achaste quem/o que me feriu.'

a-V-o [V-rel.pass $]$ Or $r_{\text {Rel }}$

(25) Kuna n-ytyka-ry

nhika-kury.

não 1SG-ver-3M.O comer-REL.F.PASS

'Não vi o que comeram / o que foi comido'

\section{$\mathrm{OR}_{\text {REL }}$ COM PREDICADO NOMINAL}

Um tipo de construção funcionalmente equivalente e também comumente usado como $O r_{\text {Rel }}$ é a simples justaposição do elemento modificado ao elemento modificador, como visto no exemplo (26), onde o predicador descritivo é interpretado como modificador do argumento do verbo da matriz:

(26) $\begin{array}{ll}\text { sytu here-ru } & \text { apu-pe } \\ \text { mulher ser.bonito-3F.O chegou-PFTV }\end{array}$

'A mulher (que é) bonita já chegou.'

Esse tipo de estrutura é comumente usado quando o foco está na simples atribuição de qualidades/propriedades ao Núc ${ }_{\text {Rel' }}$ sem que haja predicação explícita dessas qualidades/propriedades. Exatamente qual a distinção semântica ou pragmática associada ao uso mais atributivo ou predicativo desses termos de propriedade é algo que ainda requer investigação.

\section{A DISTRIBUIÇÃO DAS OR}

Ao analisarmos a posição da $O r_{\text {Rel }}$ em relação ao Núc Rel (ou vice-versa), há três possibilidades lógicas: a $O r_{\text {Rel }}$ pode preceder, suceder ou estar distribuída (descontinuamente) em ambos os lados do Núc $\mathrm{Rel}^{\prime}$ Os termos prenominal, posnominal e circumnominal são, respectivamente, associados a cada um desses tipos distribucionais (KEENAN, 1985; COMRIE, 1981/1989).

Como atestam os dados até aqui apresentados, as $\mathrm{Or}_{\text {Rel }}$ posnominais são o tipo mais comum e, de certo modo, constituem a distribuição não marcada da $O r_{\text {Rel' }}$, já que também são o tipo normalmente oferecido 
pelos falantes ${ }^{7}$. O tipo prenominal é também atestado tanto em textos como em material elicitado, como ilustram as frases em (27). Em (27a) sytu atama-nany-ta-kary 'que estava olhando para a mulher' é a $\mathrm{Or}_{\text {Rel }}$ que precede o Núc $c_{\text {Rel }}$ kyky 'homem'. Em (27b), a Or $r_{\text {Rel }}$ é pitha atama-ta-kytu 'que tu viste' que precede o Núc $c_{\text {Rel }}$ sytu 'mulher':
[ $\mathrm{O} \quad \mathrm{V}$-REL.S
a. sytu
]Or ${ }_{\text {Rel }}$
$\mathrm{S} / \mathrm{S}$
kyky
$\mathrm{V}$
mulher
etama-nany-ta-kary
homem
apu-pe
'O homem que gosta de olhar para a mulher já chegou.'
chegar-PFTV

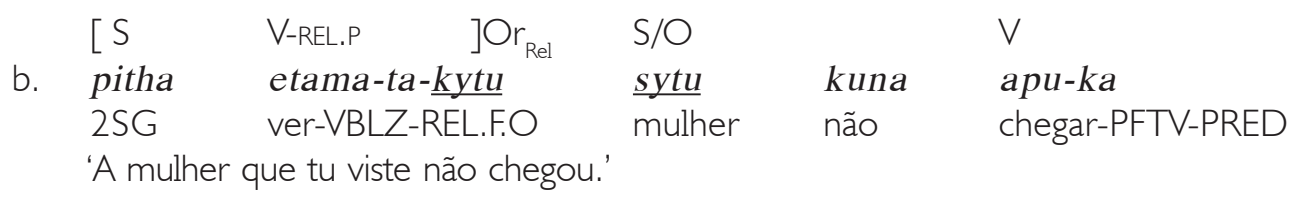

O tipo prenominal é de uso mais limitado e, por isso, mais marcado. Isso pode ser confirmado tanto pela freqüência de uso em textos quanto pelo julgamento de gramaticalidade dos falantes. Os falantes são sistematicamente mais incertos ao avaliar a gramaticalidade do tipo prenominal, ainda que este ocorra (com pouca freqüência) em textos produzidos espontaneamente. Sendo forte a tendência de interpretar o SN inicial da matriz como sujeito em frases complexas contendo $O r_{\text {Rel' }}$ é natural que o tipo pós-nominal seja mais comum (FACUNDES, 2000a).

O tipo circumnominal, em que o Núc $c_{\text {Rel }}$ ocorre internamente na $\mathrm{Or}_{\text {Rel }}$, é apenas marginalmente aceitável em Apurinã. Nas frases do exemplo (28), o Núc $c_{\text {Rel }}$ é kyky (onde os colchetes indicam a sua posição intercalada) e ocorre encaixado entre o objeto (sytu) e o verbo da $\mathrm{Or}_{\text {Rel }}$ (karuta-kary em (28a) e atama-nany-ta-kary em (28b):

$\left[\mathrm{S} / \mathrm{O} \quad\{\mathrm{S}\} \quad \mathrm{V}^{\mathrm{RELL} . \mathrm{S}}\right] \mathrm{Or}_{\mathrm{Rel}} \mathrm{V}$
a. *? sytu kyky
karuta-kary
apu-pe-ka
mulher homem ferir-REL.M.S chegar-PFTV-PRED
?'O homem que feriu a mulher já chegou.'
$[\mathrm{S} / \mathrm{O}$
$\{\mathrm{S}\}$
V-REL.S
]Or $r_{\text {Rel }}$
b. ***stu
kyky
atama-nany-ta-kary
$\mathrm{V}$
homem ver-PROG-VBLZ-REL.F.S
apu-pe-ka
( $O$ homem que estava olhando para a mulher já chegou)

Portanto, Apurinã admite os tipos de Or $_{\text {Rel }}$ pré ou pós-nominal, sendo que o último é a forma não marcada. Quanto ao tipo circumnominal, este é praticamente não gramatical na língua, já que tem sido atestado somente em dados elicitados e nem todos os falantes aceitam tal construção.

\footnotetext{
7 A possibilidade de que o padrão pós-nominal posse ser influenciado pelo português não encontra respaldo na gramática Apurinã, já que não há outra evidência de interferência da sintaxe do português na fala dos indivíduos fluentes em Apurinã, exceto possivelmente na freqüência de uso das ordens envolvendo sujeito, verbo e objeto na oração matriz.
} 


\section{O SISTEMA MORFOLÓGICO 'QUADRIPARTIDO' DE RELATIVIZADORES}

Tendo descrito as propriedades gramaticais de cada um dos tipos de $O r_{\text {Rel }}$ encontrados na língua, é possível então representar de forma mais detalhada o sistema de marcadores pronominais relativos na Tabela 2. A primeira coluna lista os tipos de $\mathrm{Or}_{\text {Rel }}$ determinadas a partir das características gramaticais dadas nas colunas seguintes: relativas com sujeito como $\mathrm{Núcl}_{\text {Rel, }}$ relativas com objeto como Núcl $\mathrm{Rel}_{\text {, }}$ relativas com passiva sem agente, e relativas com voz inversa. A segunda coluna contém a única forma recorrente em todas as $\mathrm{Or}_{\text {Rel }}$ morfologicamente marcadas, $-k$. A terceira coluna lista, respectivamente, os marcadores de voz ativa (acumulando a marcação da relação gramatical - sujeito ou objeto - do Núc $c_{\text {Rel }}$ ), voz passiva sem agente e voz inversa. $\mathrm{Na}$ quarta coluna tem-se os morfemas que podem marcar cumulativamente polaridade e número. Finalmente, na quinta coluna, há os morfemas para os gêneros masculino e feminino. As principais distinções das Or $_{\text {Rel }}$ podem ser descritas em termos de Voz, Relação Gramatical Nuclear, Polaridade \& Número e Gênero, produzindo, portanto, um grupo morfêmico quadripartido, incluindo o elemento recorrente nas relativas morfologicamente marcadas, $-k$. Ou seja, ao mesmo tempo que os marcadores pronominais relativos são segmentáveis em quatro classes morfológicas posicionais (Relativizador, Voz, Polaridade \& Número e Gênero), estes marcadores agrupam-se em torno de uma função sintática geral como marcadores de $\mathrm{Or}_{\mathrm{Rel}}$

Tabela 2. Sistema de marcadores pronominais relativos (revisada).

\begin{tabular}{|c|c|c|c|c|c|c|c|c|c|c|c|}
\hline \multirow[t]{3}{*}{ Tipos de $\mathrm{Or}_{\text {Rel }}$} & \multirow[t]{3}{*}{ Relativizador } & \multicolumn{4}{|c|}{ Voz } & \multicolumn{4}{|c|}{ Polaridade \& Número } & \multicolumn{2}{|c|}{ Gênero } \\
\hline & & \multicolumn{2}{|c|}{ Ativa } & \multirow[t]{2}{*}{ Passiva } & \multirow[t]{2}{*}{ 'Inversa' } & \multicolumn{2}{|c|}{ Positiva } & \multicolumn{2}{|c|}{ Negativa } & & \\
\hline & & Suj. & Obj. & & & SG & $\mathrm{PL}$ & SG & $\mathrm{PL}$ & $M$ & $F$ \\
\hline $\begin{array}{l}\text { Suj Núc } \\
\text { Obj Núc } \\
\text { Passiva } \\
\text { Pel } \\
\text { 'Inversa' }\end{array}$ & $-k$ & $-a$ & $-u$ & $-O$ & $-e$ & $\begin{array}{l}-r \\
-t \\
-r\end{array}$ & $-n$ & $\begin{array}{l}-t \\
-r\end{array}$ & $-n$ & $-y$ & $-u$ \\
\hline
\end{tabular}

Quando o status funcional desse grupo morfêmico quadripartido é comparado às maneiras como as línguas geralmente marcam as $\mathrm{Or}_{\text {Rel, }}$, pode-se notar que eles apresentam algumas similaridades aos pronomes relativos comumente encontrados em línguas indo-européias (KEENAN, 1985; COMRIE, 1991/1989; GIVÓN, 1989/1990). Como os pronomes relativos em outras línguas, marcadores pronominais relativos em Apurinã ocorrem dentro da $O r_{\text {Rel }}$ indicando o papel do Núc $c_{\text {Rel }}$ e funcionando como subordinadores.

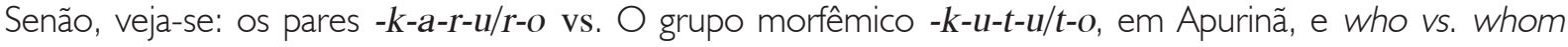
(distinção já obsoleta) em inglês, são ambos formas pronominais que ocorrem dentro da $\mathrm{Or}_{\text {Rel }}$, marcando o Núc $C_{\text {Rel }}$ como sujeito e objeto, respectivamente. As diferenças entre tais pares estão no seu status fonológico (marcas pronominais presas em Apurinã vs. marcas pronominais livres em inglês), no número de propriedades funcionais codificadas (relação gramatical, voz, polaridade, número e gênero em Apurinã vs. apenas relação gramatical e humano/não humano em inglês), na sua posição dentro da $\operatorname{Or}_{\text {Rel }}$ (após o verbo em Apurinã vs. inicial nas Or $_{\text {Rel }}$ em inglês), e, finalmente, em que apenas em Apurinã tais formas relativizadoras podem também marcar $\mathrm{Or}_{\text {Rel }}$ sem Núc $\mathrm{Rel}^{\text {. }}$

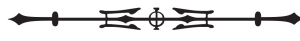




\section{ABREVIATURAS}

\begin{tabular}{|c|c|c|c|}
\hline \multicolumn{4}{|c|}{ As abreviações usadas são as seguintes: } \\
\hline $\mathrm{AFET}=$ afetado & $\mathrm{INV}=$ inversão & PFTV $=$ perfectivo & $\mathrm{PROG}=$ progressivo \\
\hline DESPOS $=$ despossuidor & INTR $=$ intransitivizador & $\mathrm{PL}=$ plural & $\mathrm{REL}=$ relativizador \\
\hline$\hat{E} N F=$ ênfase & $M=$ masculino & $\mathrm{PN}=$ predicado nominal & $\mathrm{SG}=$ singular \\
\hline $\mathrm{F}=$ feminino & $N=$ nome & POSSDO = possuído & SUJ, S, s- = sujeito \\
\hline HIPOT = hipotético & $\mathrm{OB}, \mathrm{O},-\mathrm{O}=$ objeto & PRED $=$ predicado & $V=$ verbo \\
\hline IMPFTV=imperfectivo & PASS $=$ passiva & $\mathrm{PRI}=$ privativo & $\mathrm{VBLZ}=$ verbalizador \\
\hline \multicolumn{2}{|c|}{ *?=marginalmente aceitável } & \multicolumn{2}{|c|}{ *** =completamente não aceitável } \\
\hline
\end{tabular}

\section{REFERÊNCIAS}

AIKHENVALD, 1999. The Arawak Family. In: DIXON; AIKHENVALD. The Amazonian Languages. Cambridge: Cambridge University Press. p. 64-106.

COMRIE, Bernard. 1981/1989. Language Universals and Linguistic Typology: Syntax and Morphology. 2. ed. Chicago: The University of Chicago Press.

DRYER, Matthew. 1986. Primary Objects, Secondary Objects and Antidative. Language, v. 62, p. 808-845.

FACUNDES, Sidney da Silva. 2000a. On Argument Expression in Apurinã (Arawak). In: VOORT, Hein van der; KERKE, Simom van de (Ed.). Indigenous languages of lowland South America. The Netherlands: research school of Asian; African, and Amerindian Studies (CNWS), Universiteit Leiden. p. 265-296.

FACUNDES, Sidney da Silva. 2000b. The Language of the Apurinã People of Brazil (maipure/arawak). Dissertation (Unpublished Ph.D) University of New York at Buffalo.

FACUNDES, Sidney da Silva. 2002. The Comparative Linguistic Methodology and its Contribution to Improve Knowledge of Arawakan. In: HILL, Jonathan; MORAES, Fernando (Ed.). Comparative Arawakan Studies: Rethinking Language Family and culture in Amazonia. Urbana and Chicago: University of Illinois Press. p. 74-96.

FACUNDES, Sidney da Silva. No prelo. Arawak Reconstruction and Internal Relationships: what do we know and how to know more? In: MOORE, D.; VAN DEN VORT, Hein (Ed.). Historical Linguistics in South America. [S.l.:s.n.]

FACUNDES, Sidi; BRANDÃO, Ana Paula B. No prelo. Estudos Comparativos do Léxico da Fauna e Flora Aruák. Boletim do Museu Paraense Emílio Goeldi, Belém.

FOLEY, William A.; VAN VALIN, Robert D. 1985. Information Packaging in the Clause. In: SHOPEN, T. (Ed.). Language Typology and Syntactic Description. Cambridge: Cambridge University Press. p. 282-364. v. 1.

GIVÓN, Talmy. 1989/1990. Syntax: a functional typological introduction. Amsterdam: John Benjamins. 2 v.

GREENBERG, Joseph H. 1966. Some universals of grammar, with particular reference to the order of meaningful elements. In: UNIVERSALS of language. Cambridge, Mass; MIT Press. In: SHOPEN, T. (Ed.). p. 73-113.

KEENAN, Edward L. 1985. Relative clauses. Language Typology and Linguistic Description. Cambridge: Cambridge University Press. p. 141-170. v. 3.

KEENAN, Edward L.; COMRIE, Bernard. 1977. Noun Phrase Acessibility and Universal Grammar. Linguistic Inquiry, v. 8, n. 1, p. 63-99.

PAYNE, David L. 1991. Classification of Maipuran (Arawakan) Languages Based on Shared Lexical Retentious. In: DERBYSHIRE, D. C.; PULLUM, G. K. Handbook of Amazonian Languages. [S.1.:s.n.]. p. 355-499. v. 3.

PICKERING, Wilbur N. 1977. Relativação em Apurinã. Brasília, DF: Summer Institute of Linguistics. p. 127-40. (Série Lingüística, 7).

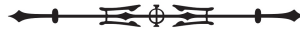

